\title{
Designing in caves: using immersive visualisations in design practice
}

Article

Published Version

Creative Commons: Attribution-Noncommercial-No Derivative Works 4.0

Open Access

Maftei, L. and Harty, C. (2015) Designing in caves: using immersive visualisations in design practice. International Journal of Architectural Research: ArchNet-IJAR, 9 (3). pp. 5375. ISSN 1938-7806 doi: https://doi.org/10.26687/archnetijar.v9i3.693 (special issue "Design Creativity: "Future Integrated Visualisation Directions") Available at https://centaur.reading.ac.uk/52849/

It is advisable to refer to the publisher's version if you intend to cite from the work. See Guidance on citing.

Published version at: https://archnet.org/publications/10416

Identification Number/DOI: https://doi.org/10.26687/archnet-ijar.v9i3.693

$<$ https://doi.org/10.26687/archnet-ijar.v9i3.693>

Publisher: ArchNet-IJAR

All outputs in CentAUR are protected by Intellectual Property Rights law, including copyright law. Copyright and IPR is retained by the creators or other copyright holders. Terms and conditions for use of this material are defined in the End User Agreement.

\section{www.reading.ac.uk/centaur}


Central Archive at the University of Reading

Reading's research outputs online 


\title{
DESIGNING IN CAVES: USING IMMERSIVE VISUALISATIONS IN DESIGN PRACTICE
}

\author{
Laura Maftei and Chris Harty \\ School of Construction Management \& Engineering, University of Reading, PO Box 129, \\ Reading, RG6 6AW, UK
}

${ }^{*}$ Corresponding Author's email address: I.maftei@pgr.reading.ac.uk

\begin{abstract}
This paper describes a study of the use of immersive Virtual reality technologies in the design of a new hospital. It uses Schön's concept of reflective practice and video-based methods to analyse the ways design teams approach and employ a full scale $3 D$ immersive environment - a CAVE - in collaborative design work. The analysis describes four themes relating to reflective practice occurring in the setting: orienting to the CAVE technology itself, orienting to the representation of the specific design within the CAVE, activities accounting for, or exploring alternatives within the design for the use and users of the space, and more strategic interactions around how to best represent the design and model to the client within the CAVE setting. The analysis also reveals some unique aspects of design work in this environment. Perhaps most significantly, rather than enhancing or adding to an existing understanding of design through paper based or non-immersive digital representations, it is often acting to challenge or surprise the participants as they experience the immersive, full scale version of their own design.
\end{abstract}

Keywords: CAVE, design teamwork, immersive virtual reality environments, reflective practice

\section{INTRODUCTION}

The combination of increasing awareness and use of Building Information Modelling (BIM) as a set of collaborative processes and developments in the availability and range of visualisation technologies accessible to practitioners is enabling new ways of performing design and construction activities. The availability of building information models has provoked new interest in the use of virtual and immersive technologies.

There is an extensive and well-established literature addressing the potential and use of virtual reality (VR) and immersive virtual reality (IVR) for design and construction. The themes addressed range broadly across technology development and piloting, experiments and case studies of use (Kahkonen, 2003). For example, Goulding and Rahimian (2011) raise attention to new social interactions and decision-making criteria enabled by such technology and indicates the potential of using VR for simulating scenarios of construction activities. This might lead to a safer environment for training AEC (Architecture, Engineering and Construction) professionals to deal with the challenges occurring in real-life situations of practice (Goulding et al., 2014) or to fostering practitioners' creativity and ability to engage with non-routine AEC activities (Rahimian et al., 2014). Another theme is integrating VR in design processes through developing and testing technology to enable more intuitive interaction with the model in the conceptual phase (Ye et al., 2006), or to better support the users' involvement in the design process (Petric et al. 2002). Feasability studies have shown how VR can support design in conceptual phase, with a focus on the impact on the cognitive and collaborative processes involved (Rahimian \&lbrahim 2011). 
Whyte et al (2000) investigated the practical use of VR technology in the industry.

This paper contributes to this literature through a concern with issues of understanding and reflecting on the effects of immersive technologies on construction design activities as used in concrete 'real-life' settings and as perceived by the practitioners involved. The setting being studied is the design of a new hospital in the UK with all patient accommodation as single rooms. There are particular client requirements around the size of the rooms, and the visibility of patients from nursing stations. Models of the single rooms were imported from CAD (Computer Aided Design) models into a CAVE -a full scale 3D immersive environment set up in a UK University lab. Over six sessions, the design teams used the CAVE to review the design against the client requirements. Adopting Schön's (e.g. 1983) perspective of reflective practice, and employing video-based methods, this paper discusses insights around the design processes occurring in the CAVE, the activity around presenting the models to the NHS (National Health Service) client and its use in demonstrating particular design requirements, and more generally around how the project teams are exploring the CAVE as a design work setting.

\section{DESIGN AS REFLECTIVE PRACTICE}

\section{Reflective practice - An overview and key concepts}

The conceptual positioning of this study is based around Schön's (1983) theory of design as 'reflective practice', which considers design as a process of both reflecting and acting, or 'thinking' and 'doing', that are inseparable and bound up in specific situations of practice. Schön positions design as a situated activity, across different individuals and materials incorporating design representations and technology. Reflective practice stresses the context dependent character of the process, accomplished through locally constructing and collaboratively sharing meanings to iteratively shape the design in responsive interaction with particular design situations. It is an open-ended process of making sense in situ, an interactive mechanism of defining and addressing, creating as well as discovering the situation. Design as 'reflective practice' is not a uniform process, but dependent on how it becomes configured in the unique, complex and messy situations of practice through both the individuals' specific ways of making sense of and approaching the situation, and through action.

It involves participants mobilising existing repertoires of knowledge, including prior understandings, and existing experiences and practices, to collectively make sense of and address particular situations. Importantly, there is an iterative relationship between what is brought in the situation to enact design work - the participants' perceptions, sense making, appreciative systems based on previous experiences and knowledge - and its outcomes which continually inform shaping of those repertoires. The media and materials in and through which the process is performed are also essential aspects of accomplishing practice. These materials cannot be disentangled or separated in their contribution to configuring and developing the process (e.g. Schön, 1983:271).

Overall, Schön argues that design as reflective practice is realised individually and collectively through reflecting and acting, drawing on appreciative systems and repertoires, and through engagement with the materials and media in the situation.

\section{Reflection-in and reflection-on action}

The essence of 'reflective practice' resides in that the 'thinking' and 'doing' are inseparable and linked through reflection which mediates enacting knowledge in response to specific situations to address the unfamiliar, unexpected, surprise instances non-approachable in routine ways.

It distinguishes two states as reflection -in and -on action. The first refers to thinking while performing the actions, and the second is a form of thinking back to action previously accomplished outside of the situation. This is a process of connecting with the understandings 
developed during action (Schön, 1992:126), enabling 'constructing an understanding' and shaping of the situation. Reflection-in-action is about understanding the situation while and through attempting to shape it. It involves thinking about the actions while performing the process. In Schön's words, the practitioner(s) is (are) reflecting-in-action "on the phenomena before him and on the prior understandings which have been implicit in his behaviour" and, in group contexts, "on the collective accomplishment of their practice and on the individual contribution to it" (Schön, 1983:68). It is configured "not necessarily through words, but through developing a 'feel for' the situation" in an "experiment of generating new understanding of the phenomenon and of changing the situation" (id.). The idea of reflection-in-action suggests the interconnectedness of perceptual mechanisms oriented to both understand and change the situation through action (Schön, 1983:134), i.e. both making sense of and shaping the design for accomplishing the process through social and material interaction.

\section{Design as reflective conversation with the materials of a design situation}

A key idea regarding design as 'reflective practice' is of understanding the process of addressing a design situation as transactional, in the sense of 'conversation' with others, and with the materials - the media and representations involved (e.g. Schön, 1984; 1991; 1992). Design is inquiry bound up in particular context through locally constructing meanings of the 'materials and messages' (other's understandings) in an ongoing process of shaping and being shaped by 'talking-back' to the situation (Schön, 1992: 126-127). This transactional, or interactional perspective on the process emphasises its situated character and the responsive, 'conversational' nature of design interactions with materials, meanings, and people, and the relationships between. 'Design as reflective conversation with the materials of a design situation' is inquiry mediated by conscious reflection on the situation and on the ways of thinking and doing employed to address it (Schön, 1992: 126). It stresses the role of the materials of a design situation in shaping the ongoing process of responding to the emergent changing states of the situation drawn on previous interventions: "Any faithful description of designing must take account of the fact that designers work in a medium [for example drawing on paper] and literally see the evolving products of their work." (Schön \& Wiggins, 1992: 154). As the transforming situation 'talks back', it brings about discovery of new meanings which will inform the further process driven by "workable understanding" (Schön, 1992:126). This is mediated through the way in which the appreciations drawn on repertoires of previous experiences are not rigid, but are also shaped during the process of attending to and accomplishing particular design events (Schön, 1983:151-152). The process of reflective conversation is stimulating further exploration. It connects with the idea of moving from exploration and initial understanding to experiencing in action, which drives continuous discovery.

An important feature in the process is responding to 'surprise' - unexpected, contradictory, unfamiliar states - mediated through 'seeing' the situation in new ways, in association with familiar elements of previous experiences, which guides the process of shaping the situation by employing action (e.g. Schön, 1983).

Other constituents interplaying with the media and the repertoires are social and material interactions. This point is described by Schön by referring to the process of engaging with the situation through 'on-the-spot' experimentation as undertaken in the 'language of designing' (1983: 80) i.e. the both verbal and non-verbal actions of orienting to the situation and to other participant's actions. Importantly, the media, language and the repertoires are inseparable in their contribution in addressing the situation: "together they make up the 'stuff' of inquiry, in terms of which the practitioners move, experiment, and explore" (Schön, 1983:271). These means of engaging with the situation - verbal and non-verbal - are essential in understanding and transforming the design: "a designer's knowing in action involves sensory, bodily knowing" (Schön, 1991: 7). This draws attention to the physicality involved in the designers' processes of 
making sense of and engaging with the situation. In relation to this point, Schön draws particular attention to the role of the media in the manipulation of 'virtual worlds' as a means of imagining, understanding and testing the design before it is actually built, and stresses the importance of how the media, language and repertoires are being manipulated for configuring a feel for the situation (Schön, 1983:157-160).

\section{The dynamics of reflection-in-action}

Schön's underlying concepts of reflecting and acting, in which the movement between the two constitutes the dynamics of the process, is described in several different ways, which can all be seen as an equivalent rehearsal of the cycle thinking-doing.

As such, the process is described as a flow of 'seeing-moving-seeing' (e.g. Schön, 1991:22; 1992), or as spiralling through stages of 'appreciation, action and reappreciation' (id., 1983:132), or as 'active sensory appreciation' (id.1991).The movement from reflecting to acting is explained as the iteration of stages of initial appreciation to formulation and realisation of intention of intervening on the situation, followed by appreciation of the consequences of previous intervention. The iteration reveals either confirmation of realisation of intention, or discovery of unintended consequences, which guides the further process by shaping the appreciations which will consequently drive following action.

Although Schön refers to the same consistent central ideas around the inseparability between reflecting and doing using different terms, often a distinction between stages appears to be implied and might be considered in tension with the core argument of reflective practice where thinking is inseparable from action. We address this point through discussing critiques of Schön's theory and by drawing awareness on this conceptual duality in terms of mobilising Schön's ideas to examine the empirical material.

Expressed by Schön in various shapes, the overall idea indicates understanding of design as ongoing process configured in iterative cycles of repeated experiments, shaped appreciative systems and understanding, or in other words through developing a 'feel for' the situation, and which, through generating cumulative discoveries, consequently inform, guide, stimulate further designing. This highlights Schön's main idea on the dynamics of the practice which is the movement between thinking and doing, mediated through reflecting-in and -on action, which enables responding to surprise, in a process of developing a 'feel for' the situation through processes of appreciation of the situation and transforming the situation in series of on-the-spot experiments.

\section{Critiques of Schön's approach of reflective practice}

As noted above, there is a possible tension between Schön's underlying idea on the inseparability between reflecting and acting, thinking and doing, and the allusion to a distinction of spiralling steps (for example seeing-moving-seeing; appreciation-action-reappreciation). The literature reflects this tension in terms of the duality by which Schön's work is both considering the process as a flow, as an artistry, configured through developing a feel for the totality of things of the situation, and in the same time, describing a structure in this process. This is related to Schön's positioning against technical rationality and instrumental accounts of practice. For example, as Roozenburg and Dorst (1998) note, "Schön turns away from Technical Rationality , but he addresses design practice without crossing into irrationality: there is a structure in design activities which can be captured from the study of what (...) practitioners do. (...) Schön does not come very far in explicating the rigour in its own terms that he ascribes to the process." (Roozenburg \& Dorst, 1998: 40).

The study recognises this tension in Schön's work with regard to understanding the process as linear versus circular, and acknowledges that this may be an attempt to demonstrate the depths of the process in a clearer way than can actually be observed in design performed in real- 
life practice. This awareness raises empirical questions around how traceable the stages of reflection-in-action are.

\section{Other studies approaching design as reflective practice}

The approach of the study builds on Schön's position as well as on more recent literature which, either by connecting broadly or by building directly on Schön's view on the process, restates the relevance of reflection in design or demonstrates the potential of mobilising reflective practice as means for understanding and analysing design work.

There is a wide range of seminal design studies connecting with Schön's ideas of reflective practice and adhering to Schön's (1983) argument around an "epistemology of practice implicit in the artistic, intuitive processes which [design and other] practitioners do bring to situations of uncertainty, instability, uniqueness and value conflict" (e.g. Cross, 2001a; 2007). This refers to reinforcing the shift from considering design as application of science to regarding it as a more complex process which is possible to be understood and explained by turning attention on the actual performance whereby the 'designerly ways of knowing, thinking and acting' are displayed to address the messy situations of practice (Cross 2001a).

The more specific ways in which these studies draw on Schön's position on design reflect their particular perspectives on the design process -for example as co-evolution of problem and solution spaces (Dorst \& Cross 2001), or as solution focused process (Lawson 2006)-, and support addressing their concerns with distinct aspects around the design process. This consists in engaging with ideas of reflective practice from an interest with understanding issues of design cognition (e.g. DTRS 6, 2003), or from a focus on the social aspects involved in team designing (Cross \& Clayburn Cross, 1995), from a concern with the use of computers in design (e.g. Lawson, 2005), to proposing theoretical models of design (Dorst \& Cross, 2001; Lawson, 2006). Within the range of studies focused on design cognition, some examine the development of design expertise (e.g. Lawson 2005; Cross 2004), or take an interest in investigating design strategies and issues of problem formulation, and generation of solutions (e.g. Cross 2001b), or draw attention on the creativity aspect (Dorst \& Cross 2001).

Among these studies broadly connected with Schön's ideas, the work of Cross (2001 b)drawn on surveying a larger set of empirical studies on design cognition- indicates that the actual performance of design is influenced by the designers' perception, construction of tasks, and by the designers' goals. Drawing on Schön's idea of framing, Cross (2001b) also points that the designers' framings -i.e. their ways of seeing particular design situations - interrelate with the designers' decisions and consequent actions.

The work of Dorst and Cross (2001) proposes a view of design as process of continuous reflection on the evolving states of problem and solution spaces, an account encapsulated in the idea of a co-evolution model of design. Making a conceptual link to Schön's idea of (problem) framing and the notion of surprise, Dorst and Cross (2001) provide an account of design not as identification of problems and then searching for satisfactory solutions, but as a more complex process of iterating between developing and refining the understanding of both problems and solutions through engaging with a task or situation. Drawing particular attention to the creative aspects of designing their empirical analysis argues that creativity is related to a situated way of defining and framing the problem, and brings about the importance of the element of 'surprise' as essential for triggering reflection-in-action. Expanding on Schön's notion of surprise as interruption of routine, Dorst and Cross (2001) suggest that a 'creative event' in design is about realising a link between the problem and solution spaces, where this link is triggered by designers' identification of surprise. Therefore surprise shapes and changes the view of the problem, enabling the seeing of things in new ways and stimulating the process.

Another way in which this broader design area of design studies connects more directly with Schön's ideas is through Lawson's work (e.g. Lawson, 2004; 2005; 2006). Lawson's argument on 
the role of perception and memory of past experiences for accomplishing a new design (e.g. 2004, 2006) is parallel to Schön's idea on how the particular ways of addressing a design situation depend on the appreciative systems and the 'repertoires' of experiences and knowledge the particular participants involved draw on. Building on another core idea of Schön's position on design as practice, specifically the understanding of design as reflective conversation with the situation, Lawson $(2005 ; 2006)$ stresses the role and the conversational or responsive nature of interactions with materials -representations and technology-, and other design participants. This presents an account of design as a process of thinking and communicating through developing representations of the design -for example by drawing.

Overall, adopting various perspectives on the process and focusing on a range of aspects around the performance of design work, these studies connect with Schön's view of design as reflective practice by considering design less as a scientific type of process, but as practical accomplishment, and realised through ongoing iteration of thinking and acting mediated through reflection. At the same time, this review finds a range of differences between the approaches adopted in these design cognition studies and the way in which this thesis takes forward Schön's conceptual position to examine design work. These differences result from their particular emphasis and focus on the cognitive aspects, arguably in detriment of showing the same attention to the 'doing' accompanying the 'thinking', and also from their tendency to aim for a generalizable understanding of the design process, potentially obscuring the situated character of design in practice.

Other studies engage more specifically with the ideas of reflective practice and mobilise related concepts in analysing empirical design situations. Some draw on Schön's theory in work examining and evaluating existing approaches to studying design (e.g. Dorst \& Dijkhuis, 1995; Dorst, 1997; Stumpf, 2001). Other studies mobilise more explicitly ideas and concepts of Schön to investigate aspects of the design process in empirical situations (e.g. Valkenburg \& Dorst, 1998; Stumpf \& McDonnell, 2002). Others build on Schön's position to develop models or frameworks for describing the design process (e.g. Dorst, 1997) and to develop methodological approaches to support the actual performance of design (e.g. McDonnell et al., 2004). These studies draw on Schön to develop insights around the team design processes and communication (e.g. Stumpf, 2001; McDonnell et al., 2004), patterns of design team behaviour (Valkenburg \& Dorst, 1998), the enactment of team thinking (Dong et al., 2013), and the use of different media in design team work (Gao \& Kvan, 2004; Rahimian \& Ibrahim, 2011; Rahimian et al., 2011).

Both limitations and advantages of mobilising Schön's approach can be seen in this literature. Dorst (1997) comments on challenges of using Schön's view in studying design, and indicates 'reflective practice' in the first form developed by Schön (1983) as a primer for a new theory (i.e. different from technical rationality), and therefore configured as "rather sketchy...and with uses...not totally clear" (Dorst, 1997:73). Nevertheless, these studies do not obscure the advantage brought by Schön's reflective practice for supporting "a description of design activities that corresponds closely to design activities as they are experienced by its practitioners", a description that appears intuitively appealing to designers (Dorst,1997: 68). As highlighted by Dorst (1997), the potential of Schön's position to link theory and practice, and to provide an approach for accessing and enabling descriptions of design "as experienced by designers" , is a quality which has been substantially addressed and developed in subsequent work, as well as in Schön's own later studies. With regard to advantages brought by drawing on Schön's position, the above studies highlight capabilities of the theory of reflective practice to access valuable insights around design processes as experienced by designers and in relation to the context, in an enriched way than possible through other paradigms, such as treating design as problemsolving process. They stress the connection between Schön's type of understanding of the process with the designers' perception of their own practice (e.g. Dorst \& Dijkhuis, 1995; Stumpf, 
2001). Overall, this literature points toward the potential of adopting Schön's view on design as reflective practice as way to access insights around a variety of aspects regarding the social, material and perceptual processes involved in the ongoing accomplishment of design activities in situ.

\section{Mobilising Schön's approach in this study}

As discussed above, the use of CAVE technology for performing design work is examined in this paper from the position of seeing design as reflective practice. The choice of adopting Schön's theoretical understanding of design as reflective practice draws on the relevance to the concern of this study, around the actual impact of immersive technology on practices of design and the effect of CAVE on design work as used in concrete 'real-life' settings and as perceived by the practitioners involved. As argued above, Schön's approach on design as reflective practice is indicated as the approach to understand and describe the process in the closest way to how it is experienced and perceived by practitioners themselves. Moreover, this position of seeing design practice as a situated activity, emphasising the interactional aspects and the role of the materials, is consistent with the empirical work in this study, which draws on observing and recording people in naturally occurring situations of practice.

Contextualising Schön's work in the broader literature indicates a wide range of seminal design studies which connect with ideas of reflective practice, considering design less as a scientific type of process, but as practical accomplishment. This is realised through ongoing iteration of thinking and acting mediated through reflection. There are similarities between more recent literature and Schön's view not least in stressing the influence of Schön's approach in the design research community by marking the shift from the previously dominant positivist perspective on design as symbolic information processing.

However, often these studies building on Schön's work take a different interpretation of Schön's view to that adopted in this paper. The more specific ways in which these studies draw on Schön's work reflect their particular perspectives on design and support addressing their specific research concerns. As such, the design cognition research community (e.g. Lawson, 1997, 2004; Cross, 2001b; Dorst \& Cross, 2001; Dong et al., 2013) highlights the more cognitive side of the process by drawing particular emphasis on the design thinking, perhaps in detriment of showing the same attention to the actions involved- the doing accompanying the thinking ${ }^{1}$. From approaches relating with behaviourally based cognitive psychology, this work draws mainly

\footnotetext{
${ }^{1}$ Schön's position on design as reflective practice treats cognition in the sense of inquiry, on Dewey's line (1938), as a "combination of mental reasoning and action in the world" (Schön, 1992:121). Differently than the design cognition research community, Schön's approach opens the door for design as practice, by turning the focus on how designers work: "In order to study reflection-in-action we must observe someone engaged in action."(Schön, 1983:322). Although some argue that "despite his emphasis on practice and experience, Schön's work is bounded by a certain cognitivist orientation" (Yanow \& Tsoukas, 2009:1343), there is a big difference between the classical cognitivist approach to design and Schön's perspective (Schön, 1983; Schön \& Wiggins, 1992) on design thinking as not contemplative, but active, by stressing the inseparability between thinking and doing in social interaction with the materials. As noted by Eastman (2001:151, 167-169, 184), Schön's work marks the shift from the classical cognitive models of design assumption on the location of design thinking in the designer's head (e.g. Lawson, 1997). Eastman also indicates Schön's description of the process as very different than the other protocol studies of design (the design cognition tradition of studies) by revealing insights in the dynamics of the process, and points out that Schön's analytical interpretation seems to correspond closely with what designers themselves think they are doing.
} 
on experimental studies conducted in artificially restricted conditions. Also, this strand of studies focused on the design thinking tends to generalise, for example through developing theoretical models, design methods and methodologies, and neglect the situated character and the context dependency of designing.

Distinct from these design cognition studies, this paper is not aiming to generalize, but to understand design as a situated phenomenon, and it is not doing experimental research and it is not focusing on the cognitive side and individual cognition aspects of the process. Schön's empirical research is mainly based on cases involving novices/ students engaged in quasiexperimental situations, rather than professionals performing actual design practice. Acknowledging this aspect, this paper differentiates between Schön's theoretical view and empirical work, and draws on Schön's conceptual understanding of design as reflective practice to focus on the social phenomenon of interacting with the immersive technology by looking at what a group of participants do in order to perform their practice in the CAVE design situation.

By focusing on the accomplishment of design work in practice, this paper resonates with the strand of studies of design as practice (e.g. Ewenstein \& Whyte, 2007; Luck, 2012). From a different perspective than the design cognition research community, these studies draw attention on the doing and reasoning involved in design as it is practiced, and turn more specific focus on the naturally occurring design interaction. However, although indicating their relation with Schön's position on design, these studies do not mobilise a reflective practice perspective, but build on other theoretical approaches and methodological commitments like, for example, organisational and aesthetics research perspectives, participatory design, ethnomethodology and conversation analysis. This study adopts Schön's ideas of reflective practice to focus on how the representations, models and technology are used and bound up in the process in a particular, specific context. Emphasising the situatedness, the interactional aspects and the role of the materials, this study employs video-based studies methods as way of mobilising the research.

In summary, this study draws on Schön's position around understanding design as realised in 'reflective conversation with a design situation' in concrete instances of practice, pointing to the contribution of particular people, representations and technology, and their inter-relationships in configuring the process through ongoing action. This literature review discussed Schön's position on design as reflective practice, acquainted his terminologies and highlighted the concepts taken forward in this research to examine how a group of design participants use a CAVE technology in a real-life empirical situation. This study mobilises Schön's ideas of design as situated, social and interactional practice-highlighting the perceptual, collaborative, communicative aspects, the role of the materials, the idea of design as conversation with the situation, and the more specific concepts of reflective practice -surprise, repertoires, appreciations, reflection-in-action, feel for the situation. In short, this refers to considering the cyclic, iterative process of reflecting and doing to address and engage with the particular design situation, and to respond to the elements of surprise by mobilising appreciations drawn on existing repertoires through both individual and collective reflection and action in conversation with the materials.

The study is also drawing attention to the movement between doing and reflecting, as well as between individual and collective reflection. At the same time, it is acknowledged that the empirical data may reveal a more fuzzy type of process, with more in-depth interlace between the phases and modes implied by Schön's model. This raises empirical questions around how traceable are the steps of the process according to Schön's model.

\section{RESEARCH METHODOLOGY}

This paper addresses the concern with understanding the effect of immersive technology on construction design activities as used in concrete 'real-life' design settings and as perceived by practitioners by drawing on and mobilising Schön's (e.g. 1983) ideas of design as 'reflective practice to examining the social phenomenon of interacting with such a technology in a particular 
situation of practice.

Schön's idea of reflective practice was developed, and explicitly positioned by Schön, as a counter to a more technical, instrumental, or objective approach to understanding professional knowledge and social practices. On this line of argument emphasising the inseparability between theory and practice, the design knowledge is in the action, in practice, and not a rigid application of science. It is not a process of following prescriptive rules, but of making sense of the unpredictable, messy, conflicting situations of practice and developing new understanding through experience in the situation. Schön' view argues that the design process can be understood through "close examination of what (...) practitioners actually do" within "unique, uncertain, and conflicted situations of practice" (Schön, 1983: viii- ix). Stressing the uniqueness of the design situations in practice, Schön's (1983) approach argues for an 'epistemology' of design as "reflective practice", idea which connects with adopting a strategy of inquiry through "reflection on the actual practice of (...) practitioners who reflect in action" (Schön, 1983:133). This research choice refers to understanding the design phenomenon through examining the practice, the actual performance of design, and to treat the actions whereby design participants perform the process as implicit to answering the research inquiry (Schön, 1983:49, 133). This approach points to accessing the dynamics of the phenomenon through examining how particular design participants think and work with representations, using technology and interacting with people in particular design settings. Consistently with the approach on design as reflective practice, the process can be understood by examining the practice through looking at what practitioners actually do in particular design situations: "A close look at a small, homely example of designing, in the narrower sense, will give us a parable of design practice" (Schön, 1992:127).

Drawing on this methodological approach, this paper operationalises the research through examining an empirical case of real-life design process performed using a CAVE. In line with the methodological principles set from the broader position of the study, the research mobilises direct observation and video-based research methods. The rational for choosing this approach builds on the consistency between the methodological principles of video-based studies - prioritising the situated and interactional accomplishment of practical action (Heath et al., 2010) - and the broad position of the study of conceptualising design as situated practice. Augmenting direct observation with video recordings draws on the tenets of video-based studies of workplaces and of interactions (Heath \& Luff, 2008; Heath et al., 2010), which recognise and emphasise the potential of using video for examining how the interplay between the talk, visible conduct and the use of artefacts becomes relevant to the accomplishment of a social activity. This standpoint stresses practical advantages in mobilising video in research to investigate the ways in which the participants of interactions such as design meetings perform their actions on reviewing the design, to explore the interplay of their interactions with and around the technology, and to examine how they orient themselves to the technology and to other participants' actions. Rather than just employing direct observation, video recording brings the practical value of capturing gestures and movements alongside the verbal interaction as well as the ability to 'replay' the data during analysis. Although not commonly practiced, there is a methodological congruence between Schön's concept of reflective practice and video-based techniques in capturing the detail of design interactions.

The case study was based on an on-going project for designing a new hospital in the UK. One of the requirements is that all patient accommodation is in single rooms, rather than traditional multi-bed wards. Single room only accommodation is rare in the UK, and so a key issue for the client was ensuring that the rooms were of sufficient size, and that visibility into rooms was adequate. At the time of the research, the project was still in tender preparation stage. The project team opted to augment traditional design and client engagement activities with the use of a CAVE - a full scale 3D immersive environment set up in a UK University lab. This was to be used to demonstrate room size and show accurate lines of sight. 
As particular type of immersive environment, the CAVE (Cave Automatic Virtual Environment) is a multi-person, room- sized, high-resolution multi-display 3D video and audio environment, in which graphics are projected onto the walls and the floor producing a fully immersive, true scale rendition. It offers the user (equipped with 3D stereo glasses and a head mounted tracking device with location sensor) active real-time interaction with a life sized 3D model. One user's movement in the space of the CAVE is being tracked and, consequently, perspective rendering is displayed responding to their position and movements (i.e. looking up or down). CAVE participants are fully within the space and can easily interact between themselves during the simulation (De Fanti et al., 2011). The CAVE at the University of Reading has three vertical projection screens ( $3 \mathrm{~m}$ by $2.2 \mathrm{~m}$ ) and a floor projection screen ( $3 \mathrm{~m}$ by $3 \mathrm{~m})$.

The research used video recording and direct observation of a series of six sessions held within the CAVE, involving project and design managers, architects and designers, and modellers and visualizers, in various combinations. These were spread across five months, between November 2011 and March 2012. These sessions produced approximately 12 hours of audiovideo recordings. Various combinations of video cameras were used to capture the design meetings: one hand-held camera, a second camera fixed on a tripod, positioned in one corner of the CAVE and a third camera fixed on the CAVE's ceiling to offer an aerial top down view. Conducting the research followed the University's ethical procedures regarding the participants' consent and the confidentiality and data protection.

\section{ANALYSING REFLECTIVE PRACTICE IN THE CAVE}

The analysis below discusses insights around the design process occurring in the CAVE, the activity around presenting the models to the NHS client and their use in demonstrating particular design requirements, and more generally around how the project teams are exploring the CAVE as a work setting. It is specifically concerned with examining how performing design reviews in the immersive environment are accomplished through situated interaction with a focus on understanding how the representations, models and technology mediate the process. Initial analysis of the video data let to the emergence of four tentative themes:

- orienting to the technology / situation;

- orienting to the design;

- thinking about the use and users of the designed space;

- representing the design to the client.

For the purposes of this paper, one approximately two minute vignette from the data set is used to unpack and demonstrate these themes and trace the relevance of Schön's insights on design practice.

\section{A fragment of the empirical material}

The video fragment below is extracted from the first of the six sessions reviewing the design in the CAVE, and refers to how the architects and contractors teams first encounter the immersive environment and perform a walkthrough of the virtual model of the hospital. This design event occurs in the first part of a one hour review session, and the interactions captured unfold between approximately minute 9 and minute 11 of this first design meeting held within the CAVE. It depicts a sequence when the project teams experience their first visit in the CAVE. The vignette is preceded by a general presentation around the technology, verbally introduced by the CAVE technical staff members. Following this, the design participants start their familiarisation with the immersive environment by removing their shoes (to protect the sensitive floor projection area), equip themselves with the 3D glasses and the head tracker, and begin to examine the state of the model in the main atrium area, in a joystick navigated simulation mode. In this stage of reviewing the design, the participants express a particular interest around the representational aspect of the CAVE rendering of the 3D model, and with how their design meets the client's 
requirements.

\begin{tabular}{|c|c|c|}
\hline Designer 2 & (09:07): & $\begin{array}{l}((\ldots)) \text { Just back (.) Can we have back a view from the reception } \\
\text { area? }\end{array}$ \\
\hline & (09:08): & There! ((pointing upwards to the right side of the displayed model)) \\
\hline & 10): & Just go back (.) a little bit left (.1) There! ((pointing to the left)) \\
\hline & (09:12): & We need to cut that back (.2) ((pointing) \\
\hline & (09:15): & $\begin{array}{l}\text { ((because })) \text { You can't see through the concourse }(.) \text { if you're sitting } \\
\text { on those chairs ((in the atrium)) }\end{array}$ \\
\hline & $(09: 17):$ & ((Designer 1 is leaning tow \\
\hline $\begin{array}{l}\text { Designer } 1 \\
\text { Designer } 2\end{array}$ & (09:19): & $\begin{array}{l}\text { So: }(.) \text { That wall? }((\text { pointing towards her left hand side }))= \\
=\text { Yes }(.) \text { There! }\end{array}$ \\
\hline Desi & $\begin{array}{l}(09: 21): \\
(09: 23):\end{array}$ & $\begin{array}{l}\text { Yea:h (.) Absolutely. } \\
\text { ((towards Designer 3)) Can you check that back ((in the office))? }\end{array}$ \\
\hline Designer 3 & $(09: 25):$ & Yeah (.2) ((making a hand note)) \\
\hline & (09.27): & $\begin{array}{l}\text { I'm not sure why }(.2) \text { I thought that it was open view ((the } \\
\text { perspective towards the concourse)) }\end{array}$ \\
\hline Designer 1 & $(09: 30):$ & $\begin{array}{l}\text { Well that's ok (.) Yeah, that's the point of looking in a 3D } \\
\text { environment (.) To see what we're actually doing! }\end{array}$ \\
\hline Designer 1 & (10.00): & $\begin{array}{l}\text { And this door (.1) it should be glazed! So you would see daylight } \\
\text { coming through! }\end{array}$ \\
\hline Designer 4 & (10:10): & $\begin{array}{l}\text { It sounds good (.) I think we should capture as much detail as we } \\
\text { can. }\end{array}$ \\
\hline $\begin{array}{l}((\text { The } t \\
\text { renderir }\end{array}$ & uss & $\begin{array}{l}\text { the CAVE technician around the possibilities of refining the CAVE } \\
\text { rease the realism of the simulation)) }\end{array}$ \\
\hline Designer 4 & $(10: 45):$ & $\begin{array}{l}\text { That's good (.) It's impressive cause }(.1) \text { This is quite good (.) cause } \\
\text { showing this perspective is something you can't work out in any } \\
\text { other way! }\end{array}$ \\
\hline & $(10: 48):$ & $\begin{array}{l}\text { To look (.1) ((points upwards to the right side of the model)) } \\
\text { To see (.) upwards ((pointing to indicate the view of the atrium from } \\
\text { ground floor up to the first floor through to the glazed curve shaped } \\
\text { roof)) }\end{array}$ \\
\hline
\end{tabular}




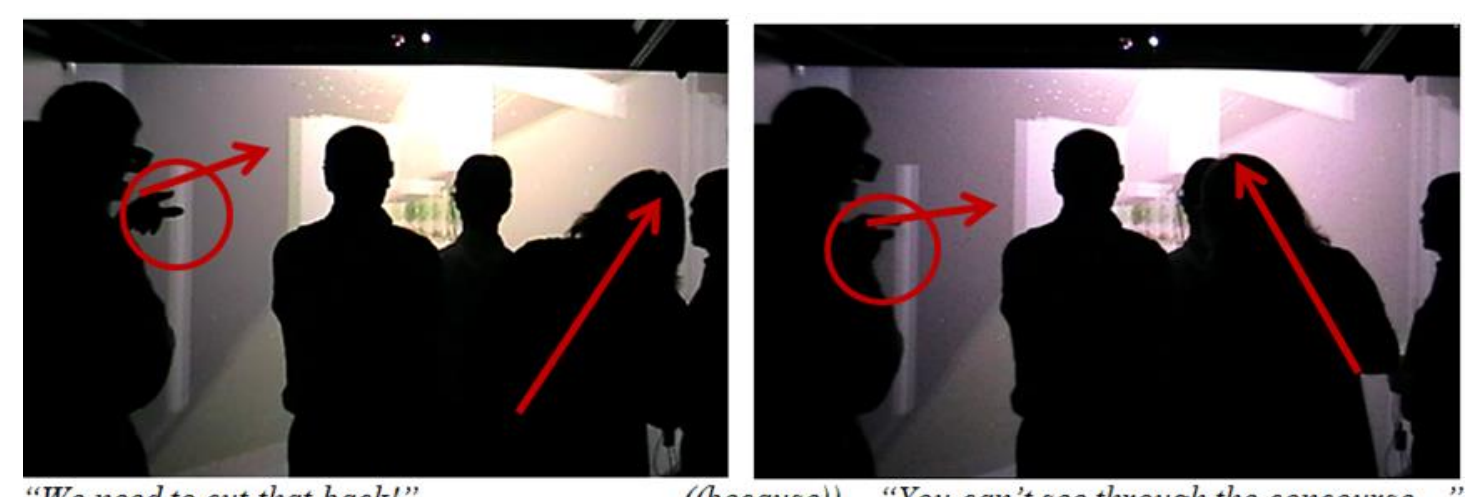

"We need to cut that back!"

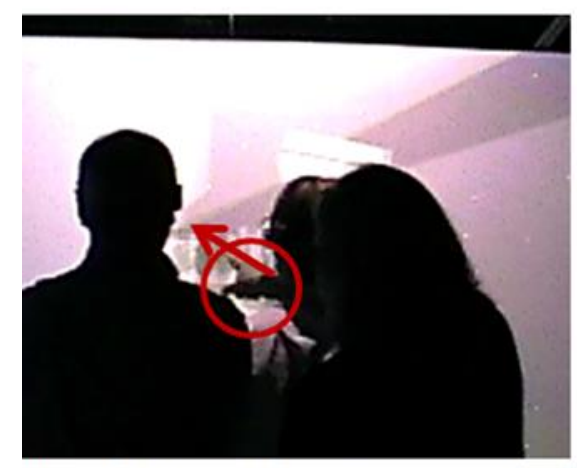

"So: (.) That wall ?(...) Can you check that?" (because)) "You can't see through the concourse..."

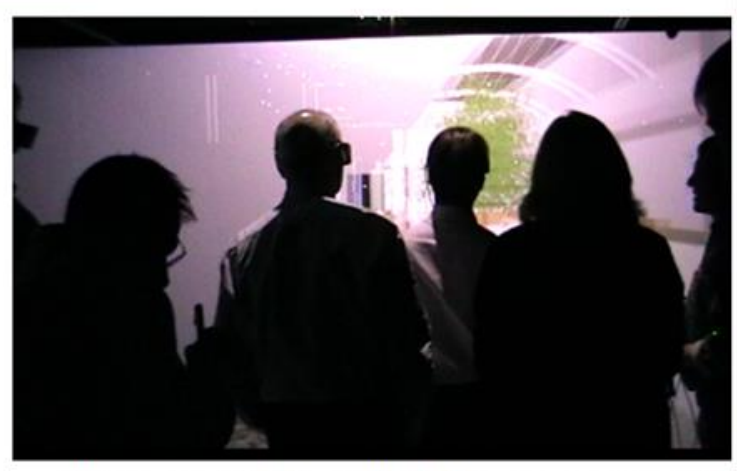

"Yeah. (but)) I thought that it was open view!"

"...that's the point of looking in a $3 D$ environment (.) To see what we're actually doing!"

Figure 1. Episode 1 (E1, 09:12-09:30)

CAVE technician

Designer 4

(11:06):

(11:00):

(11:01):
If you get down, actually you can see a bit better

((The contractor wearing the head trackers starts lowering down his body towards a kneeling position))

((General loud laughing from the other participants))

You don't have to!

((leaning down and extending his arm to support the kneeling contractor))

((General laugh continues))

((The viewing perspective of the model changes, offering a more ascendant image towards the roof from the eye level of a person sitting down on a chair))

Contractor

(11:22):

CAVE technician

Designer 2

(11:24):

(11:28):

CAVE technician
So if you move through, you can see what's there!

((gradually standing up))

Yeah.

If we wanted to model daylight coming through that space, can we see it?

Yeah (.) you can do that $((\ldots))$ but would be easier to model scenes of clouds, or blue sky (...) 

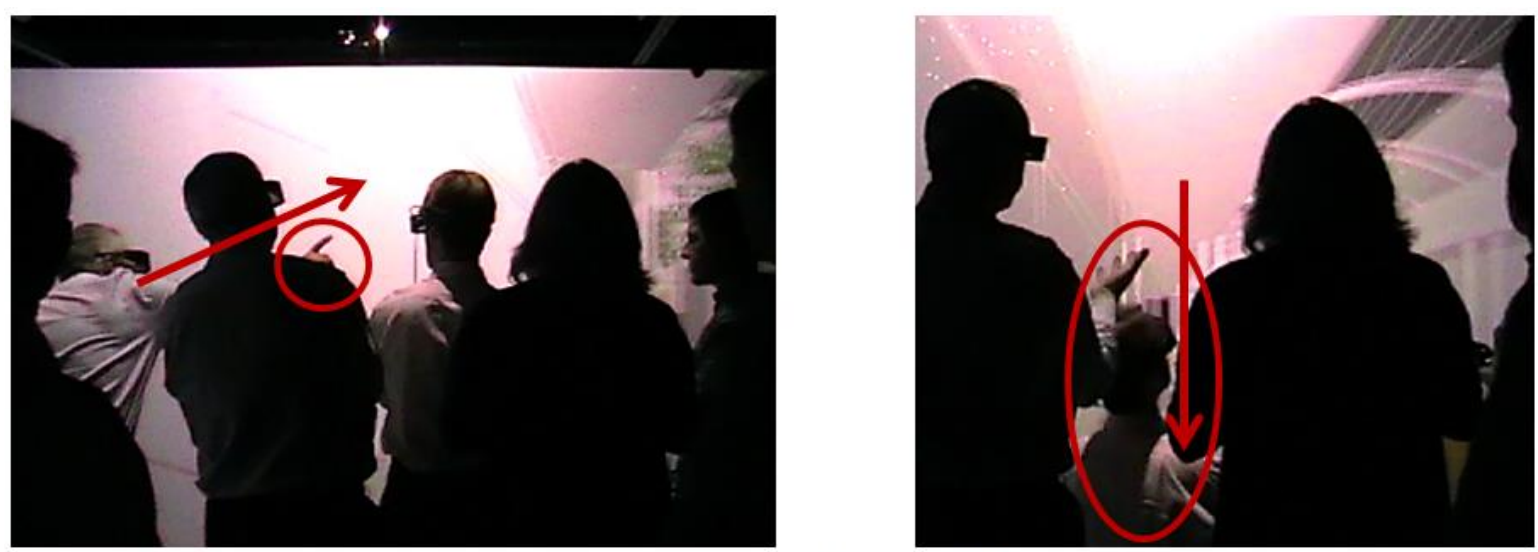

“(...) this perspective (...) you can't work out in any other way!"

"If you get down (...) you can see a bit better"
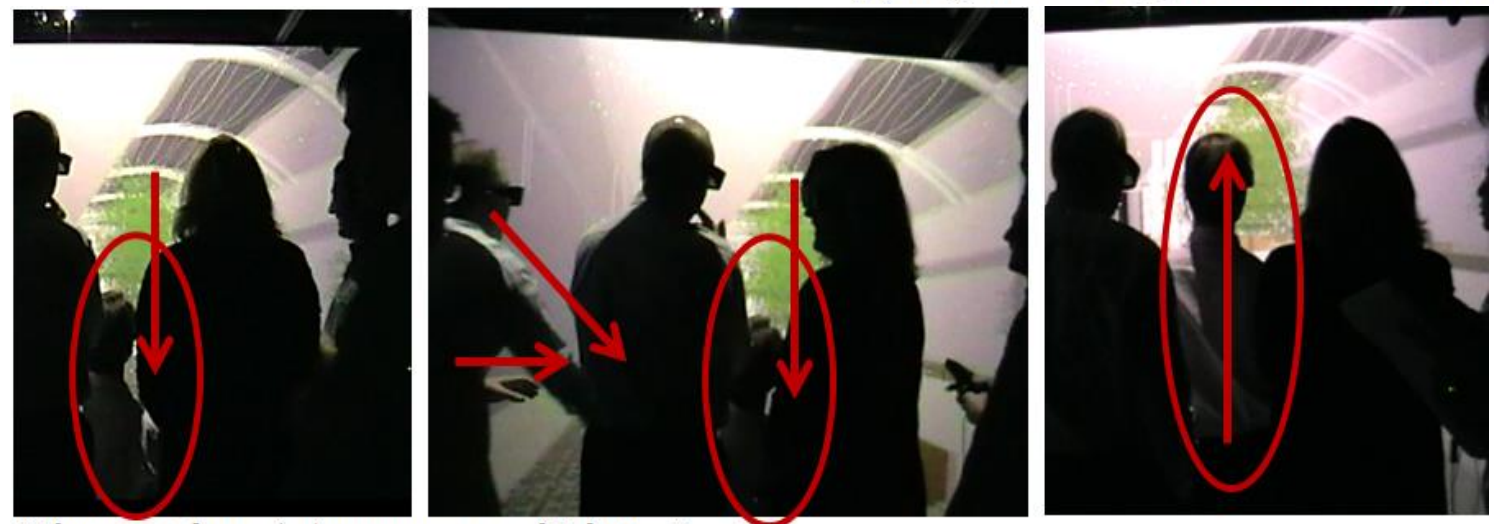

"If you get down (...) you can see a bit better"

"You don't have to!"

"So if you move through, you can see what's there!"

Figure 2. Episode 1 (E1, 10:45-11:22)

\section{Exploring the situation}

In the moments preceding the interactions in the fragment presented above, the design and contractor teams were focusing on aspects of visual quality of the representation of the 3D model in the CAVE. The meeting was organized around the participants' discussion with the CAVE technical staff members around the technology, in terms of capabilities and limitations of the immersive environment, particularly with regard to displaying lighting and shadows, and materials and materiality (textures, colours, mapping) in the CAVE rendering.

Orienting to the design and to the technology (E1, 09:07- 09:10)

"Just back! Can we have back a view from the reception area?"

Starting with the instance opening this episode (E1), the design review process is disrupted by the reaction of Designer 2 towards a spontaneously perceived misfit in the design of the reception area: "Just back! Can we have back a view from the reception area?". Although the broader concern of the meeting was around the visualisation features of the CAVE representation, the simulation of the hospital building triggers Designer 2's attention on the state of the design scheme. Noticing an unexpected issue about the design, the participant asks the CAVE technician (who is navigating the model by joystick) to shift the visualisation of the model in an area previously displayed, and engages the other team members to re-examine the design of the atrium. The misfit regards the lack of confirming one of the design requirements in the model, 
more specifically the issue of enabling visibility from the reception area towards the two levels open space of the atrium, up to the transparent curve shaped roof.

\section{Orienting to the design by accounting for the use and users (E1, 09:12- 09:21)}

"You can't see through the concourse ...if you're sitting on those chairs"

Moreover, this lack of conformity between the form and function of their design (the limitation of the scheme in terms of meeting the requirements) is considered with regard to the use of the design as real building, from the perspective of the future users: "You can't see through the concourse ... if you're sitting on those chairs" ((in the atrium)).

This episode of discovering the design clash consequently triggers their decision on applying further changes to the design by removing the wall that was blocking the visibility:

"We need to cut that back"

This intention of changing the design formulated by Designer 2 is subsequently confirmed by other team members ("Yeah"; "Absolutely") through a series of group interactions around establishing a shared understanding of the model in the CAVE.

In Schön's terms, noticing the unexpected non-conformity of the model with the client's requirement is an element of surprise perceived in the design, surprise which triggers the designer's reflection on the situation with regard to changing the scheme. The instance denotes also a circumstance of moving, as a formulation and realisation of a design intention in response to the feedback of the representation. This sequence unfolds through a process of collaborative reflection-in-action whereby the participants manage to make sense together of the design, and to establish a shared understanding and group decision around further developing the design, with regard to addressing the discussed area of the interior space of the hospital. This is realised through the participants' orientation to both the design, and also to the technological setting, through a series of actions configured by interplaying verbal and bodily interactions among each other and with the display of the design (as captured in Fig.1), and whereby members of contractor and design teams align their vision as a group.

Moving between orienting to the technology and to the design (E1, 09:08- 09:21)

"There!" (...) "Just go back ... a little bit left ... There!" (...) "So: That wall?"

This process is collaboratively accomplished through firstly identifying the element blocking the visibility among multiple participants, each having different viewing perspective and hence different perception of the projection of the 3D model. This is due to their various locations in the space of the CAVE. The sequence denotes partly their reflection on the others' understandings, and partly how, for addressing this constraint in visualising the model in the same way in the CAVE, the group employs a set of situated interactions of orienting to the technology. This process is mediated through both verbal and bodily behaviour whereby the participants develop a shared vision on the design: between the moments $E 1,09: 08$ and $E 1,09: 21$ the review process is supported by the concerted actions of Designer 2, Designer 1 and the Cave technician. These interactions are expressed through verbal indications -like, for example, "There"; "A bit to the left"-, gestures -like pointing and arm movements to indicate the element-, multiple changes of gaze, or adjustments of bodily position, orientation or height (such as in the sequences below). 

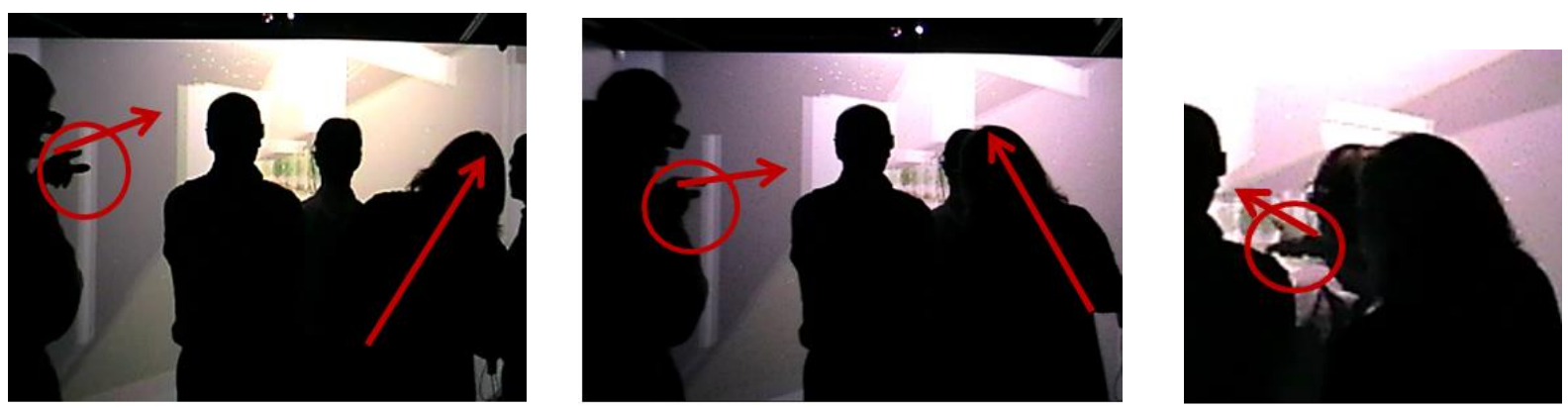

"There!" ((pointing upwards to the right side of the displayed model))

"Just go back ... a little bit left ... There!" ((pointing to the left))

"Yes (.) There!"

"So: That wall?" ((pointing towards her left hand side))

Figure 3. Episode 1 (E1, 09:08-09:19)

The data above exemplify the interplay of verbal with non-verbal interactions within making sense of the design situation in the CAVE: Designer 1 shifts her body orientation from leaning towards the right to the left side of the CAVE. Concurring to the situation, the Cave technician orients the joystick navigation of the model according to the verbal and bodily indications of Designer 2. The sequence illustrates the participants' processes of addressing the design situation through iteratively orienting to the technology and shaping an understanding of the CAVE rendering of the model and of the scheme.

Also, the sequence infers a process of moving between making sense of and engaging in conversation with the design situation in the CAVE. The participants iterate between orienting to the unfamiliar technological setting, making sense of the CAVE version of the design, and in response to the understandings gradually developed around the current state of the design, drawing collaborative decisions regarding further changes to the scheme and refinement of the representation.

Orienting to the design and representing: changing the design (E1, 09:12- 09:25)

"We need to cut that back!"

Designer 2 (09:12):

We need to cut that back (.2) ((pointing))

(09:15):

((because)) You can't see through the concourse (.) if you're sitting

(09:17):

on those chairs ((in the atrium))

(09:19):

Designer 1

((Designer 1 is leaning towards left $))$

Designer 2

Designer 1

So: (.) That wall? ((pointing towards her left hand side $))=$

$=$ Yes $($.$) There!$

(09:21):

Yea:h (.) Absolutely.

These interactions immediately following the previous sequence in the episode reveal an emerging movement from the participants' shared understanding of the model to addressing the situation by formulating the intention of changing the design : "We need to cut that back!" (Designer 2) and establishing the group agreement around the decision of changing the design "Absolutely!". Consequently, between the moments E1, 9:23 and E1, 09:25, the flow of this process of orienting to their design leads the participants to move from understanding the model and deciding changes of removing the wall blocking the visibility towards the atrium to making steps towards actually materialising this decision. This is realised through collaborative interaction between Designer 1 and Designer 3 around further applying the established intervention on the design outside the CAVE, in the architects' offices. The interaction is verbally expressed by Designer 1- "Can you check that back?" and followed by the confirmation "Yeah" of Designer 3 who is also marking a hand-note about the design change in his notebook. 


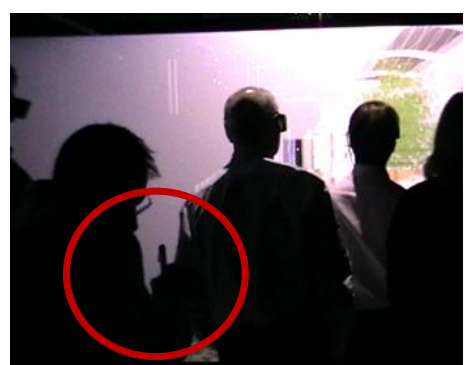

Figure 4. Episode $1(\mathrm{E} 1,09: 25)$

In Schön's perspective, the sequence may be referred to as moving from formulation of intention to realising the intention of changing the design situation. Also, the sequence already marks a shift from using the CAVE in the review process not only as a presentation tool, but also as a medium which contributes by enriching the designers' understanding and informing the further development of the scheme itself.

\section{Orienting to the design: not confirming expectations (E1, 09:27) \\ "I'm not sure why ... I thought that it was open view"}

The previous sequence is immediately accompanied by the Designer 3's reflection on the design scheme, on the representation and on the medium: "I'm not sure why ... I thought that it was open view". The Designer 3's reaction restates the surprise perceived around the clashing element in the design and infers that drawing on his previous experience of visualising the model (on paper, Revit on a computer screen) his assumption was that the visibility requirement towards the concourse had already been addressed in the design. In Schön's vocabulary, the sequence infers that the design element blocking the visibility in the atrium is perceived as unexpected from Designer 3's appreciation system, drawing on his repertoire of understanding. Also, from a 'reflective practice' perspective, the sequence exhibits a circumstance in which the feedback of the representation reveals a lack of conformity with the design expectations, and consequently triggers reflection on the previous and current actions, understandings, media and procedures, accompanied by generating new meanings and employing new action to address the situation in a different way.

Orienting to the technology (E1, 09:30)

"(...) that's the point of looking in a 3D environment (...) To see what we're actually doing!"

These interactions of orienting to the design, are entangled subsequently with Designer 1's reflection on the use of the medium to support design practice: "...that's the point of looking in a 3D environment ...To see what we're actually doing!". The designer expresses an awareness developed around how visualising and experiencing the model in the CAVE supports the designers' collaborative appreciation of the outcome of former design intentions, and enables the project teams to notice issues about the design which were unexpected from previous representations. Interestingly, the last aspect indicates a circumstance in which the process of orienting to the design in the CAVE environment is challenging previous understanding drawn on the usual design procedures based on using other types representations and technology.

Representing the design: drawing attention to the detail (E1, 10:00-10:45)

"(...) I think we should capture as much detail as we can"

Further in the episode, Designer 3's reflection on the role of the media for revealing issues about the design triggers drawing attention to the level of detail of the representation in the CAVE:

"And this door should be glazed! So you would see daylight coming through!" (E1, 10:00).

The moment marks a circumstance of orienting to the design by noticing another unconformity with the requirements - the door should be transparent - from the concern with the 
experience of the space by potential users -"you would see daylight". It is a transition in the focus of the meeting from understanding the scheme to starting to think about more detailed representational aspects. Consequently, the design participants shift the focus of the meeting to the potential of using the CAVE for enhancing the representation. A fourth participant, Designer 4 finds the idea of actually seeing daylight through the particular window in the CAVE simulation of the model as a useful detail for providing a real like experience of the virtual design. This instance triggers the participants' attention to thinking about representing the design for the client: "It sounds good. I think we should capture as much detail as we can" (E1, 10:10). Subsequently in the sequence, the team discusses with the CAVE technician around the possibilities of refining the CAVE rendering of the design for increasing the realism of the simulation.

Orienting to the technology (E1, 10:45- 10:48)

'It's impressive (...) cause showing this perspective is something you can't work out in any other way"

Following this, the participants move again to thinking about the potential of the particular immersive environment to support the review process in ways not possible using other types of design media: "It's impressive...cause showing this perspective is something you can't work out in any other way" (E1, 10:45). Designer 4's reflection expands on the initial familiarisation with the ways of navigating the model in the CAVE, by moving towards exploring the potential of the medium to support their process going forward. Building up an understanding of the technology, the participants suggest advantages of the CAVE through enabling an immersive experience of being inside the life size scale virtual model of the design with own physical body: "...to look..((panoramically)) ... to see upwards!". "It's impressive $((\ldots))$ cause showing this perspective is something you can't work out in any other way! To look ((points upwards to the right side of the model)) To see (.) upwards"

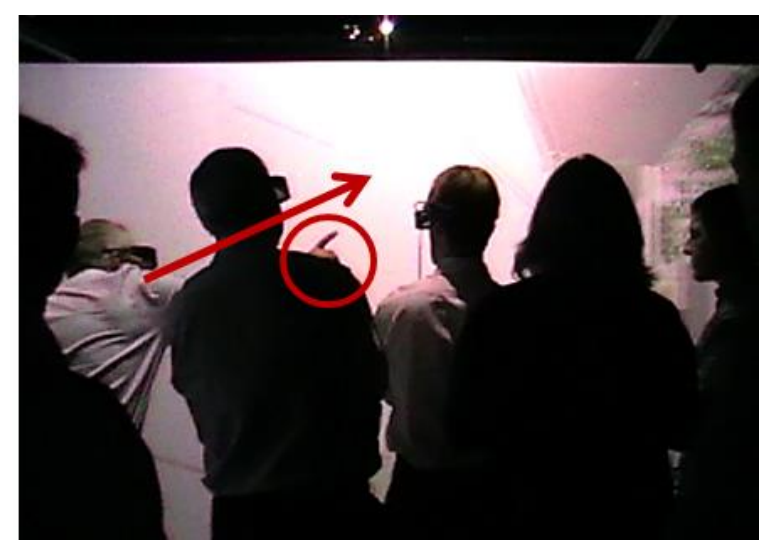

Figure 5. Episode 1 (E1, 10:45- 10:48)

Throughout their process of gradually familiarising themselves with the CAVE technology in the course of the review session, the participants are shaping an understanding around the potential of using the immersive setting for performing design. This type of process illustrates what in Schön's vocabulary might be referred to as developing a "workable understanding" (Schön, 1992:126), or a 'feel for' the design situation and for the medium. These aspects are observable in how, starting the moment E1, 11:00, they begin to orient to the technology through exploring the type of experiencing and the ways of understanding the virtual model in the CAVE. The design participants' familiarisation with the technology earlier described is mainly drawn on verbal introduction around the technology from the CAVE technical staff around informing them about particularities of using the CAVE, such as taking off the shoes for protecting the floor projection area, equipping with the head trackers and special 3D glasses to achieve stereo view and active navigation. In the beginning of the episode, although one of the participants is already wearing the head tracker, the navigation is still lead by joystick by the CAVE technician who simulates the model through the key areas in the model according to verbal guidance of the project teams. But here they are beginning to explore the possibilities of the model, moving beyond their initial orientation. 
Orienting to the technology: making sense of the potential of the technology in use "If you get down, actually you can see a bit better" (E1, 11:00-11:05)

Starting the instance E1, 11:00, when the Contractor wearing the head tracker is actually kneeling to test the CAVE technician's suggestion of getting lower to then look up, the participants begin to make sense of the capability of the CAVE to enable a more dynamic simulation of the walkthrough, and recognise their own body movements in responsive interaction with the virtual model. The sequence exhibits rich verbal and bodily behaviour of the participants. They note how, in response to the Contractor's body movement and change of height, the images of the rendering displayed on the projection screen change accordingly, offering a more ascendant view towards the roof of the atrium from the eye level of a person sitting down on a chair in the reception area. This whole episode is accompanied by a general tone of amusement, marked by loud laughing within the teams' members, and by the reaction of one participant (Designer 4) to spontaneously initiate a gesture of trying to help the Contractor get back to a standing position. This is expressed by Designer 4 through the verbal utterance "You don't have to!", augmented by a bodily behaviour of leaning down towards the kneeling Contractor and extends his arm to support him.

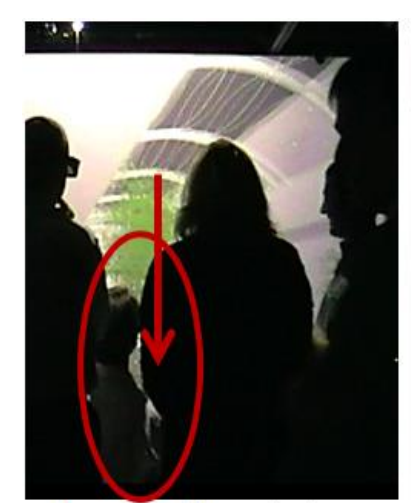

"If you get down, actually you can see a bit better"

"You don't have to!"

"So if you move through, you can see what's there!"

Figure 6. Episode 1 (E1, 11:00- 11:12)

This reaction indicates how the group perceives the particular type of interaction with the technology and with the model in a design meeting as different and unexpected. The event denotes the surprise perceived around the unfamiliar way of experiencing a 3D simulation of design, which is new to them. The sequence reveals a particular type of social interaction emerging within performing design review in the CAVE environment, both in terms of a sense of fun and excitement, and through bringing a range of non-verbal means of interacting among group members.

This connects with a process of orienting to, or making sense of the use of technology, an aspect which in Schön's vocabulary is developing "workable understanding" (Schön, 1992:126), or a feel for the medium and for the ways of doing design in the CAVE, through shaping a group understanding around the potential of the immersive environment. The fragment displays how the participants develop a feel for the possibilities of enhancing the representation and the CAVE experience of the design for impressing the client. This gradual process of orienting to the technology is observable in the sequential movement from "...you would see daylight coming through" (E1, 10:00), to "It sounds good (.) I think we should capture as much detail as we can." (E1, 10:10); "That's good (.) It's impressive cause ... showing this perspective is something you can't work out in any other way!"; "to see upwards" (E1,10:45), and then, towards the final part of 
the fragment, to thinking about modelling daylight to show it in the CAVE simulation (E1, 11:28, 11:29), and so on. The data conveys a sense of the continuity and flow of this situated process, realised through iteration between episodes of orienting to the technology, making sense of the design and thinking about further developing the scheme and the model, and about representing it for the client.

\section{DISCUSSION AND CONTRIBUTIONS}

From the analysis, four themes or sets of activities can be identified. There is initially some collaborative effort made to make sense of this novel environment and to establish what it can do, for instance in the conversations with the technician around levels of detail or the function of the head-tracker (e.g. E1, 09:07- 09:10, or E1, 11:00-11:29). This is orienting to the CAVE technology. Simultaneously, the participants are collectively making sense of the representation of the design within the environment (e.g. E1, 09:07-09:30), through asking for different viewpoints (E1, 09:07-09:10), moving around the space (e.g. E1, 11:00-11:22) and noticing aspects of the design they did not expect from their prior understanding of it. For example, by simulating the model in the CAVE, the participants discover that their design scheme is not complying with the client requirement around the visibility towards the reception area $(E 1,09: 15)$. This is perceived as surprising because it is not confirming their expectations drawn from previous visualisations of the design- REVIT on screen (E1, 09:27). The surprising outcome is subsequently triggering the participants' decisions on further change the design $(E 1,09: 12)$. In this case they comment that the environment can show perspectives on the design that other media cannot ( $E 1,09: 30, E 1,10: 45-10: 48)$. This can be seen as orienting to the specific design within the CAVE. Just a few seconds later, the team is not just discussing aspects of the design, but the perception of it from the point of view of users, such as rehearsing the view from the chairs in the atrium (E1,09:15), or discussing the glazing in the door to increase the life-like nature of the model (E1, 10:10-10:45). This is exploring alternatives for the use and users of the space. The discussions also go beyond the design brief and use to the way it can be demonstrated to the client, for instance in modelling the sky, clouds and external features (e.g. $\mathrm{E} 1,10: 10-10: 45$; or E1,11:28-11:29). This can be seen as strategic interactions around how to best represent the design and model to the client.

Together, these four themes of 1, orienting to the CAVE technology itself, 2, orienting to the representation of the specific design within the CAVE, 3, activities accounting for, or exploring alternatives within the design for the use and users of the space, and 4, more strategic interactions around how to best represent the design and model to the client within the CAVE setting represent a pattern of engaging with design work in this immersive, novel environment.

\section{The dynamics of reflective practice}

The empirical material also illustrates that these four analytical themes overlap, even in a short fragment of two minutes of interaction. The findings indicate that the process of reviewing the design in the CAVE follows an iterative flow of orienting to the technology and to the design, thinking about the use/ users of the space, and representing out the design for showing it to their client. In line with Schön's perspective, this type of empirical process is revealing a pattern of reflective practice, through spiraling between discovering and designing, making sense of the situation and actively addressing it, in a flow of both shaping the situation and, in return, shaping the understandings and the subsequent actions.

Reflecting on the debate over the dynamics of reflective practice, the study confirms a tension between linearity versus a circularity of the process. The analysis of the video data identifies the steps by which the participants perform the process in situ through orienting to the technology, making sense of the design, actually doing design work, then thinking about the use and users, and about representing the design for engaging with the client. The thematic analysis 
shows that these steps can be identified and focused upon separately, but it also reveals a circularity and simultaneity or concurrence of these, in the entanglement by which these types of processes occur concomitantly, and without following a specific order. Relating back to the critique discussed above, the data shows exactly the fuzziness indicated by the studies criticising Schön. Drawing on the empirical findings, the study indicates that these thematic aspects are addressed both in distinct stages, and intertwined, revealing a complex, interdependent and iterative process in which making sense and design work are inseparable. Therefore the study confirms the tension around the linearity versus circularity of the design process and recognises this issue in the empirical data as a complex combination of processes.

\section{Other insights to Schön's approach to understanding design practice}

The study contributes to Schön's approach to understanding design practice by accounting for, and analysing in detail the non-verbal interaction through bringing together Schön's approach on design as reflective practice and a video-based studies approach as method for inquiry. The findings of this study reveal some unique aspects of design work in this immersive environment, that span across and beyond these analytical themes:

- The data suggests distinct social aspects of design behaviour emerging in the CAVE,

especially with regard to the participants' excitement (amusement, episodes of laughing) in the environment.

- The physical and bodily presence of the design participants within the CAVE model is

connected to a greater reflection on ways of using the space, and even simulating the roles and actions of end users.

- Performing design in the CAVE environment is enhancing or adding to an existing

understanding of design through paper based or non-immersive digital representations, and perhaps most significantly, it is also challenging or surprising the participants as they experience the immersive, full scale version of their own design.

Overall, the findings of the study demonstrate the relevance of mobilising Schön's approach to design as situated, social, material, and perceptual practice, and the more specific concepts of reflective practice - surprise, repertoires, appreciations, reflection-in-action, feel for the situationas a useful way of inquiring the use of the CAVE in performing design work. The study also demonstrates the utility of video-based data collection techniques for capturing the details of design interactions. Although this is not commonly used in design oriented studies, in this case it offered a useful and practical means to retain and replay the data, and potentially offers an alternative or supplementary method to further explore the contours of design practice.

The study enhances the existing understanding around the practical consequences of using CAVE's in design, and informing the development of the technology from a practice perspective. Distinctively, these contributions build on and extend prevailing work in the existing usability testing and technology development literature.

\section{CONCLUSION}

The aim of this study was to examine the use and the implications of immersive virtual reality technologies in the construction design process. This aim was addressed by taking an interest in the use and implications on the actual design practice and practitioners, and it was pursued through reflecting on the effect of immersive technologies on construction design activities as used in concrete 'real-life' settings and as perceived by the practitioners involved. Empirically, this broader aim was investigated by focusing on the use of CAVE technology in a real world design project, from a perspective stressing the participants' view on the role and utility of the technology in performing actual design work.

The central idea highlighted through the study points out that using the CAVE both mediated and bounded up in performing design work configured as social, collaborative and context 
dependent process. The study found that, moving beyond initial intention of using the technology as a better way to convince the client within the bid process, the participants used the CAVE for more than just presenting the design, but also for performing actual design work.

The study clearly identified a complex set of interactions emerging in the CAVE design process through the participants' iteration between collaboratively making sense of the technology, orienting to the design in this environment, and then doing further design work in consequence to perceiving new issues or finding out unexpected aspects about their design. Importantly, the CAVE design review experience did not only provide a better representation of the 3D model, in a more exciting design environment, and it did not only extend, but it also challenged participants' understandings of their design and their usual ways of working. The findings suggest that the immersion in the life-sized version of the 3D model, the feel of space, the physicality involved and the social aspect of designing in the CAVE, contributed to distinct ways of accomplishing design practice. Performing design in the CAVE was realised in a fundamentally social and interactive way, by making sense of the technology and of the CAVE representation of the design, interacting with the model and among participants, and then doing more design work. Using the CAVE for reviewing the design triggered changes to the design and affected the further development of the project and the engagement with the client.

\section{Limitations and potential avenues for further research}

There are limitations to all research. This research was limited to investigating a particular design phase (design review, part of the bid preparation) within a particular project. This empirical context enabled a close examination of the detailed interaction occurring in the design situation of the particular design episodes, but it would have been interesting to follow the use of the CAVE in other stages in the process, such as for early design. A potential avenue for further work might be to investigate how the CAVE would be used earlier in the process, where there is less certainty around the design, as well as later, in detailed design and construction phases. It might also be interesting to examine the use of the CAVE as design setting for developing other projects, involving other design teams, and to trace possible patterns of design interactions across multiple situations.

Capturing the video data encountered several technical limitations, particularly caused by the lighting issues in the CAVE, but also by the way of setting the video cameras. The study experimented various combinations of cameras- fixed on tripod placed in the corners of the CAVE, fixed mounted on the ceiling for aerial view, and mobile hand held cameras. Using more fixed cameras would allow better capturing the detail of gestures.

In relation to the findings around the surprise and challenge triggered by the use of the CAVE on designers' understandings and procedures, the study indicates that an interesting potential avenue for further work would be to examine the implications of integrating the CAVE within design usual work and procedures. Future research could examine how the practitioners would think about and use the CAVE if they would have the technology in their daily workspaces and how would this change the dynamics of their practice.

\section{ACKNOWLEDGEMENTS}

This study is based on a PhD research undertaken at the University of Reading as part of, and funded through, an EPSRC (Engineering and Physical Sciences Research Council) centre on Healthcare Infrastructure (HaClRIC - Health and Care Infrastructure Research Centre).

\section{REFERENCES}

Cross, N. \& Clayburn Cross, A. (1995). Observation of teamwork and social processes in design. Design Studies, 16(2), 143-170.

Cross, N. (2001a). Designerly ways of Knowing: Design Discipline versus Design Science. Design Issues, 17(3), 49-55. URL: http://www.jstor.org/stable/1511801 
Cross, N. (2001b). Design cognition: results from protocol and other empirical studies of design activity. In C. Eastman, W. Newstatter \& M. McCracken (Eds.), Design knowing and learning: cognition in design education (pp. 79-103). Oxford, UK: Elsevier.

Cross, N. (2004). Expertise in design: an overview. Design Studies, 25, 427-441. doi:10.1016/j.destud.2004.06.002

Cross, N. (2007). Forty years of design research. Design Studies, 28(1), 1-4. doi: 10.1016/j.destud.2006.11.004

Dorst, K. \& Cross, N. (2001). Creativity in the design process: co-evolution of problem-solution. Design Studies, 22, 425-437.

De Fanti, T. A., Sandin, D., et al. (2011). The future of the CAVE. Central European Journal of Engineering, 1(1), 16-37.

Dong, A., Kleinsmann, M. S. \& Deken, F. (2013). Investigating design cognition in the construction and enactment of team mental models. Design Studies 34, 1-33. doi:10.1016/j.destud.2012.05.003

Dorst, K. \& Dijkhuis, J. (1995). Comparing paradigms for describing design activity. Design Studies, 16: 261-274.

Dorst, K. (1997). Describing design: a comparison of paradigms. Technische Universiteit Delft. Rotterdam. PhD Thesis.

DTTRS6 (Design Thinking Research Symposia) (2003). Cross, N. and Edmonds, E. (Eds.). Expertise in Design. Creativity and Cognition Press, University of Technology, Sydney, Australia.

Eastman, C. M. (2001). New Directions in Design Cognition: Studies of Representation and Recall. Design Knowing and Learning: Cognition in Design Education. C. M. Eastman, W. M. McCracken and W. C. Newstetter, Elsevier Ltd: 147-198.

Ewenstein, B. and J. Whyte (2007). Visual representations as 'artefacts of knowing'. Building Research \& Information, 35(1): 81- 89.

Gao, S. \& Kvan, T. (2004). An Analysis of Problem Framing in Multiple Settings. Design Computing and Cognition '04, 117-124.

Goulding, J. S., \& Pour Rahimian, F. (2011, November 3-5, 2011 ). A Game-Like Virtual Reality Construction Site Simulator for Non-Collocated Collaboration in AEC Environments. Paper presented at the 11th International Conference on Construction Applications of Virtual Reality 2011 (CONVR 2011), The University of Bauhaus.

Goulding, J. S., Pour Rahimian, F., \& Wang, X. (2014). Virtual reality-based cloud BIM platform

for integrated AEC projects. Journal of Information Technology in Construction (ITCON), 19(Special Issue BIM Cloud-Based Technology in the AEC Sector: Present Status and Future Trends), 308-325.

Heath, C., Hindmarsh, J., \& Luff, P., Eds. (2010). Video in Qualitative Research. Analysing Social Interaction in Everyday Life. Introducing Qualitative Methods. London: SAGE Publications Ltd.

Heath, C. \& Luff, P. (2008). Video and the Analysis of Work and Interaction. In P. Alasuutari, L. Bickman \& J. Brannen (Eds.), The Sage Handbook of Social Research Methods (pp. 493-505). London: Sage Publications Ltd.

Kahkonen, K. (2003). Editorial: Virtual Reality Technology in Architecture and Construction. Journal of Information Technology in Construction (ITCon), 8: 101- 103.

Lawson, B. (2004). Schemata, Gambitis and Precedent: Some Factors in Design Expertise. Design Studies, 25(5), 443- 457. doi:10.1016/j.destud.2004.05.001

Lawson, B. (2006). How designers think. The design process demystified, Oxford, UK:Architectural Press.

Lawson, B. (2005). Oracles, draughtsmen, and agents: the nature of knowledge and creativity in design and the role of IT. Automation in Construction, 14, 383-391. doi:10.1016/j.autcon.2004.08.005

Luck, R. (2012). Kinds of seeing and spatial reasoning: Examining user participation at an architectural event. Design Studies 33(6): 557-588.

McDonnell, J., Lloyd, P., \& Valkenburg, R. C. (2004). Developing design expertise through the construction of video stories. Design Studies, 25(5), 509-525. doi:10.1016/j.destud.2004.05.005

Petric, J., Maver, T., Conti, G., \& Ucelli, G. (2002). Virtual reality in the service of user participation in architecture. Paper presented at the CIB W78 Conference, Aarhus School of Architecture.

Rahimian, F. P., Ibrahim, R., Rahmat, R. W. B. O. K., Abdullah, M. T. B., \& Jaafar, M. S. B. H. (2011). Mediating Cognitive Transformation with VR 3D Sketching During Conceptual Architectural Design Process. Archnet-IJAR, International Journal of Architectural Research, 5(1), 99-113.

Rahimian, F. P., \& Ibrahim, R. (2011). Impacts of VR 3D sketching on novice designers' spatial cognition in 
collaborative conceptual architectural design. Design Studies, 32(3), 255-291.

Rahimian, F.P., Arciszewski, T., \& Goulding, J. S. (2014). Successful education for AEC professionals: case study of applying immersive game-like virtual reality interfaces. Visualization in Engineering, 2(4). doi:10.1186/2213-7459-2-4

Roozenburg, N. \& Dorst, K. (1998). Describing design as reflective practice: Observations on Schön's theory of practice. In E. Frankeberger, P. Badke-Schaub \& H. Birkhofer (Eds.), Designers, the key to successful product development (pp. 29-41). London, UK: Springer.

Schön, D. (1983). The reflective practitioner. How professionals think in action. London: Temple Smith.

Schön, D. (1984). The Architectural Design Studio as an Exemplar of Education for Reflection-in-Action. Journal of Architectural Education, 38(1), 2-9. URL: http://www.jstor.org/stable/1424770

Schön, D. (1991). Designing as reflective conversation with the materials of a design situation. Keynote talk Conference on Artificial Intelligence in Design. Edinburgh, UK, 25th June 1991.

Schön, D. (1992). The theory of Inquiry: Dewey's Legacy to Education. Curriculum Inquiry, 22(2), 119-139. URL: http://www.jstor.org/stable/1180029

Schön, D. \& Wiggins, G. (1992). Kinds of seeing and their functions in designing. Design Studies, 13(2), 135-156.

Stumpf, S. (2001). Analysis and representation of rhetorical construction of understanding in design teams' experiential learning, University College London, PhD Thesis.

Stumpf, S. \& McDonnell, J. (2002). Talking about team framing: using argumentation to analyse and support experiential learning in early design episodes. Design Studies, 23, 5-23.

Valkenburg, R. \& Dorst, K. (1998).The reflective practice of design teams. Design Studies, 19, 249-271.

Yanow, D. \& H. Tsoukas (2009). What is Reflection-In-Action? A Phenomenological Account. Journal of Management Studies 46(8): 1339-1364.

Ye, J., Campbell, R. I., Page, T., \& Badni, K. S. (2006). An investigation into the implementation of virtual reality technologies in support of conceptual design. Design Studies, 27(1), 77-97.

Whyte, J. (2002). Virtual Reality and the built environment. Oxford, Architectural Press.

Whyte, J., Bouchlaghem, N., Thorpe, A., \& McCaffer, R. (2000). From CAD to virtual reality: modelling approaches, data exchange and interactive 3D building design tools. Automation in Construction, 10(1), 43.55.

\title{
AUTHORS
}

\author{
Laura Maftei \\ School of Construction Management \& Engineering, \\ University of Reading \\ I.maftei@pgr.reading.ac.uk
}

\section{Chris Harty}

School of Construction Management \& Engineering,

University of Reading

c.f.harty@reading.ac.uk 